\title{
Hyperdiploid with Status of Chromosomes 4 and 10 Unknown
}

National Cancer Institute

\section{Source}

National Cancer Institute. Hyperdiploid with Status of Chromosomes 4 and 10 Unknown. NCl Thesaurus. Code C124873.

Having a chromosomal number greater than the normal diploid number, with the status of chromosomes 4 and 10 unknown. 